\title{
Valutazione economica dei costi associati al trattamento di pazienti in ossigenoterapia a lungo termine, con o senza monitoraggio telemetrico domiciliare
}

Roberto Ravasio*, Roberto W. Dal Negro ${ }^{\circ}$, Carlo Lucioni*

\begin{abstract}
Long term oxygen treatment (LTOT) represents an helpful, even though costly, therapeutic strategy for managing severe chronic respiratory diseases at home. The aim of the present study was to assess the economic impact of adding the home telemetric monitoring of some vital signs to the traditional domiciliary LTOT in this kind of patients.
\end{abstract}

Two samples of severe chronic respiratory patients (COPD patients in $89 \%$ of cases, managed at home without, $\mathrm{n}=20$, and with, $\mathrm{n}=61$, telemetric home monitoring) were compared for a 24 -month period, and the corresponding outcomes measured. In the tele-monitored group of subjects, both the mean number and the mean duration of hospitalisations dropped along the two-year study, together with the n. exacerbations/ patient/year. The mean annual cost for the tele-monitored group of patients was lower by $28 \%$ in the first year, and by $33 \%$ in the second year of the study.

The home tele-LTOT management of patients with severe chronic respiratory disease, mostly COPD, allows a better clinical control of the disease, with a corresponding $50 \%$ reduction of exacerbations leading to hospitalisation.

Finally, the home tele-LTOT contributes substantially in minimizing the economic impact of these severe chronic respiratory diseases.

Keywords: long term oxygen treatment (LTOT), COPD, home telemetric monitoring, pharmacoeconomics, cost of illness

Farmeconomia e percorsi terapeutici 2005; 6 (4): 349-352

\section{PREMESSA}

I limiti evidenti nella disponibilità delle risorse che caratterizzano i sistemi sanitari dei paesi economicamente sviluppati impongono di effettuare delle scelte terapeutiche non solo in funzione della rilevanza clinica, ma anche della convenienza economica delle alternative disponibili. A questo fine la semplice considerazione del costo di acquisto di un trattamento non è sufficiente, ma occorre considerare il consumo complessivo di risorse indotto da una determinata scelta terapeutica.

Gli studi di valutazione economica tendono oggi a focalizzarsi principalmente su tecnologie rilevanti, senza dare troppo spazio ai settori di nicchia, ovvero a quei beni e servizi che, come ad esempio l'ossigenoterapia, sem- brano non produrre grossi volumi di spesa o che comunque riguardano patologie così dette "non emozionali" agli occhi degli organi decisori [1].

Questa valutazione rappresenta una eccezione a questa tendenza, perché riguarda appunto l'ossigenoterapia a lungo termine (OTLT) [2-3]. L'ossigenoterapia è stata introdotta in Europa nei primi anni ' 80 principalmente per il trattamento della broncopneumopatia cronica ostruttiva (BPCO), e in generale, per tutte le patologie polmonari [4-5].

Per valutare appieno l'importanza economica di questa terapia si può citare un recente studio [6] che ha analizzato i costi di trattamento della $\mathrm{BPCO}$ e ha evidenziato come in realtà il costo dell'ossigenoterapia domiciliare appaia elevato, raggiungendo il $30,2 \%$ di
* Wolters Kluwer Health, Adis International ltd., Milano

- U.O.C. di

Pneumologia

ULSS22 Regione Veneto

Ospedale Orlandi,

Bussolengo, Verona 


\section{Tabella I}

Numero medio e durata ricoveri ospedalieri (24 mesi di osservazione)

\begin{tabular}{lccccc}
\hline \multirow{2}{*}{ Descrizione } & \multicolumn{5}{c}{ Monitoraggio telemetrico } \\
\cline { 2 - 5 } & \multicolumn{2}{c}{ Primo Anno } & \multicolumn{2}{c}{ Secondo Anno } \\
\cline { 2 - 5 } & sì & no & sì & no \\
\hline Numero medio ricoveri per pz. & 0,7 & 1,6 & 0,5 & 1,5 \\
Durata media degenza (gg) & 11,6 & 21,0 & 7,4 & 20,9 \\
\hline
\end{tabular}

tutti i costi diretti sanitari, con un costo medio per paziente di circa 1.400 euro all'anno. Per inciso, lo stesso studio ha rilevato che l'ossigenoterapia viene praticata dal $40 \%$ circa dei pazienti con BPCO in fase di mantenimento, contro il $14 \%$ dei pazienti in fase di riacutizzazione.

Obiettivo di questo studio è la valutazione dell'impatto economico conseguente al monitoraggio telemetrico domiciliare dell'ossigenoterapia in pazienti in trattamento a lungo termine verso pazienti in ossigenoterapia senza monitoraggio telemetrico.

\section{MATERIALIE METODI}

Lo studio utilizza i dati provenienti da due coorti di pazienti seguiti dalla Divisione di Pneumologia dell'Ospedale di Bussolengo (Verona):

- 1995 (gruppo storico, $\mathrm{n}=61$ ), pazienti con ossigenoterapia a lungo termine e telemonitoraggio domiciliare

- 2003 (gruppo di controllo, $n=20$ ), pazienti con solo ossigenoterapia a lungo termine.

Le due coorti sono state seguite per un periodo di osservazione di due anni. La valutazione è stata condotta in base alla prospettiva del Servizio Sanitario Nazionale. I costi sono stati valorizzati al 2004

Le informazioni raccolte per i due gruppi di pazienti riguardano la diagnosi dei pazienti e il numero medio annuo di riacutizzazioni con conseguente ricovero ospedaliero.

I pazienti di entrambi i gruppi erano affetti da patologie polmonari, in prevalenza da $\operatorname{BPCO}(89 \%)$.

Per quanto riguarda il consumo di risorse e la relativa valorizzazione dei costi legati alla somministrazione della ossigenoterapia di lun-

\begin{tabular}{lcc}
\hline \multicolumn{1}{c}{ Descrizione } & $\mathbf{1 9 9 5}$ & $\mathbf{2 0 0 4}$ \\
\hline Gas analisi & 0,62 & 1,64 \\
$\mathrm{O}_{2}(1,5 \mathrm{~L} / \mathrm{m}$ in $\times 18 \mathrm{~h} /$ day $)$ & 6,66 & 7,39 \\
Personale med.-inferm. & 1,86 & 2,3 \\
Farmaci & 0,64 & 1,05 \\
Monitoraggio telemetrico & 0,21 & 0,41 \\
\hline
\end{tabular}

\section{Tabella II}

Costi medi giornalieri per OTLT go termine con o senza monitoraggio telemetrico domiciliare, si è fatto riferimento ad uno studio pubblicato nel 1995 [7] che ha calcolato un costo medio giornaliero per l'OTLT sulla base delle seguenti componenti: gas analisi, ossigenoterapia, personale medicoinfermieristico, farmaci concomitanti e monitoraggio telemetrico.

Il costo delle riacutizzazioni con ricovero ospedaliero è stato derivato da uno studio di Lucioni et al [8].

\section{RISULTATI}

La Tabella I mostra il numero medio annuo di riacutizzazioni con conseguente ricovero ospedaliero per le due coorti considerate, rilevate nel primo e nel secondo anno di osservazione.

Da questi dati si evince come il numero medio di ricoveri per paziente e la durata media della degenza di ogni singolo ricovero risultino sensibilmente inferiori per la coorte 1995 (monitorata con telemetria domiciliare) rispetto a quella 2003. In particolare, nel primo anno di confronto, per la coorte 2003 il numero medio annuo per paziente di riacutizzazioni con ricovero è di 1,6 mentre per la coorte 1995 tale valore scende a $0,7(-56,3 \%)$. Questa differenza aumenta (-66,7\%) se si analizzano i dati relativi al secondo anno di osservazione $(1,5$ vs 0,5$)$.

\section{Il costo del trattamento domiciliare}

La Tabella II, ripresa dallo studio di Micheletto et al. [7], mostra i valori originali del 1995 e i valori attualizzati (inflated) al 2004 (indice ISTAT) dei costi medi giornalieri associati alla somministrazione dell'ossigenoterapia domiciliare: gas analisi, ossigenoterapia, personale medico-infermieristico, farmaci concomitanti e monitoraggio.

Sulla scorta di questi risultati è stato calcolato un costo medio giornaliero sia per i pazienti con ossigenoterapia a lungo termine e monitoraggio telemetrico pari a 12,79 euro e sia per i pazienti con solo ossigenoterapia a lungo termine pari a 12,38 euro (Tabella III).

In entrambi i casi il principale driver di costo è rappresentato dall'ossigeno che spiega quasi il $60 \%$ del costo medio totale. La seconda voce di costo in ordine di importanza è rappresentata dal personale medico-infermieristico con un'incidenza pari a circa il $13 \%$. La diffe- 
R. Ravasio, Dal Negro RW, C. Lucioni

renza tra $\mathrm{i}$ due costi medi giornalieri di 0,41 euro $(+3,3 \%)$ è costituita dal costo medio giornaliero imputato al servizio telematico necessario per effettuare la telemetria.

E stato poi calcolato un costo medio annuo per paziente, pari a 4.668,35 euro (gruppo 1995) e a 4.518 .70 euro (gruppo 2003), moltiplicando l'importo giornaliero per 365 giornate.

\section{Il costo delle riacutizzazioni ospedalizzate}

Lo studio di Lucioni et al [8] ha calcolato che a più del $90 \%$ dei pazienti dimessi da ricovero dopo riacutizzazione (BPCO) vengono assegnati due DRG: il DRG 088 (Malattia polmonare cronica ostruttiva) $(69,6 \%)$ oppure il DRG 087 (Edema polmonare e insufficienza respiratoria) $(23,6 \%)$. Alla restante parte dei ricoveri è stato assegnato un DRG compreso in una decina di possibili altri (Tabella IV).

In base alla distribuzione dei DRG e delle rispettive tariffe di rimborso è stato calcolato un costo medio ponderato per ciascun ricovero dovuto a riacutizzazione di BPCO pari a 3.218 euro (Tabella V).

Moltiplicando il costo medio ponderato per DRG per il numero medio di riacutizzazioni con ricovero è stato calcolato il costo medio annuo per le riacutizzazioni trattate con ricovero ospedaliero rispettivamente per la coorte 1995 e per la coorte 2003 nel primo e nel secondo anno di osservazione (Tabella VI).

Infine sono stati calcolati i costi medi annui totali di trattamento (OTLT più riacutizzazioni) per i due gruppi di pazienti considerati.

Nel primo anno di osservazione il gruppo di pazienti con monitoraggio telemetrico domiciliare è caratterizzato da un costo medio annuo inferiore a quello senza telemonitoraggio domiciliare (28\%). Tale differenza aumenta nel secondo anno di osservazione (33\%).

Questo risparmio, pari a $2.746,55$ euro e a 3.068,35 euro nel primo e nel secondo anno rispettivamente, nonostante la presenza di un più alto costo medio per la gestione del moni-

\begin{tabular}{lcccc}
\multicolumn{1}{c}{ Descrizione } & \multicolumn{2}{c}{$\begin{array}{c}\text { Con } \\
\text { teleossimetria }\end{array}$} & \multicolumn{2}{c}{$\begin{array}{c}\text { Senza } \\
\text { teleossimetria }\end{array}$} \\
\hline Gas analisi & 1,64 & $12,82 \%$ & 1,64 & $13,25 \%$ \\
$\mathrm{O}_{2}(1,5 \mathrm{~L} / \mathrm{m}$ in $\times$ 18h/day $)$ & 7,39 & $57,78 \%$ & 7,39 & $59,69 \%$ \\
Personale med.-inferm. & 2,3 & $17,98 \%$ & 2,3 & $18,58 \%$ \\
Farmaci & 1,05 & $8,21 \%$ & 1,05 & $8,48 \%$ \\
Monitoraggio telemetrico & 0,41 & $3,21 \%$ & 0 & $0,00 \%$ \\
Costo medio die & $\mathbf{1 2 , 7 9}$ & $\mathbf{1 0 0} \%$ & $\mathbf{1 2 , 3 8}$ & $\mathbf{1 0 0 \%}$ \\
\hline
\end{tabular}

Tabella III

Costo medio (in euro) die per paziente OTLT con o senza teleossimetria (2004)

\begin{tabular}{clcc}
\hline \multirow{2}{*}{ Codice DRG } & \multicolumn{1}{c}{ Descrizione DRG } & N. & $\%$ \\
\cline { 2 - 4 } 088 & Malattia polmonare cronica ostruttiva & 500 & $69,5 \%$ \\
087 & $\begin{array}{l}\text { Edema polmonare e insufficienza } \\
\text { respiratoria }\end{array}$ & 169 & $23,5 \%$ \\
475 & $\begin{array}{l}\text { Diagnosi relative all'apparato respiratorio } \\
\text { con respirazione assistita }\end{array}$ & 13 & $1,8 \%$ \\
089 & $\begin{array}{l}\text { Polmonite semplice e pleurite, età > } \\
\text { con complicanze }\end{array}$ & 9 & $1,3 \%$ \\
144 & $\begin{array}{l}\text { Altre diagnosi relative all'apparato } \\
\text { circolatorio con complicanze }\end{array}$ & 7 & $1,0 \%$ \\
Altro & $\quad 21$ & $2,9 \%$ \\
Totale & & 719 & $100 \%$ \\
\hline
\end{tabular}

Tabella IV

Distribuzione dei DRG

\begin{tabular}{cc}
\hline Costo del ricovero secondo i DRG & Tutti i DRG \\
\hline N. & 719 \\
Media & 3.218 \\
Min & 601 \\
Max & 96.398 \\
\hline
\end{tabular}

Tabella V

Costo medio ponderato DRG

\begin{tabular}{ccccc}
\hline \multirow{2}{*}{ Descrizione } & \multicolumn{4}{c}{ Monitoraggio telemetrico } \\
\cline { 2 - 5 } & \multicolumn{2}{c}{ Primo Anno } & \multicolumn{2}{c}{ Secondo Anno } \\
\cline { 2 - 5 } & sì & no & sì & no \\
\hline Costo riacutizzazioni in ricovero & $2.252,60$ & $5.148,80$ & $1.609,00$ & $4.827,00$ \\
\hline
\end{tabular}

\begin{tabular}{lcc|cc}
\hline & \multicolumn{4}{c}{ Monitoraggio telemetrico } \\
\cline { 2 - 5 } \multicolumn{1}{c}{ Descrizione } & \multicolumn{2}{c}{ Primo Anno } & \multicolumn{2}{c}{ Secondo Anno } \\
\cline { 2 - 5 } & \multicolumn{1}{c}{ sì } & no & sì & no \\
\hline Costo OTLT & $4.668,35$ & $4.518,70$ & $4.668,35$ & $4.518,70$ \\
Costo riacutizzazioni in ricovero & $2.252,60$ & $5.148,80$ & $1.609,00$ & $4.827,00$ \\
Costo medio totale di trattamento & $6.920,95$ & $9.667,50$ & $6.277,35$ & $9.345,70$ \\
\hline
\end{tabular}

\section{Tabella VI}

Costi medio annuo per paziente per le riacutizzazioni trattate con ricovero (in euro)

\section{Tabella VII \\ Costi medi annui totali} (in euro) 
toraggio (4.668,35 euro vs 4.518,70 euro), è generato dal minor costo medio dovuto alla minor incidenza di riacutizzazioni ospedalizzate (Tabella VII).

\section{CONCLUSIONI}

L'esercizio di valutazione economica qui condotto consente di concludere che l'utilizzo del monitoraggio telemetrico domiciliare nei pazienti con ossigenoterapia a lungo termine permette un miglior controllo della patologia, riducendo di oltre il 50\% il numero delle riacutizzazione con ricovero, con una diminuzione della spesa sostenuta dal Servizio Sanitario Nazionale per la gestione di questo tipo di pazienti.

Gli Autori dichiarano che lo studio è stato condotto senza alcuna sponsorship.

\section{BIBLIOGRAFIA}

1. Mantovani L. Quadro teorico di riferimento in "L'intervento privato in sanità" Garattini L (a cura di), Milano, Kailash Editore, 1995

2. Garattini L, Tediosi F. L'ossigenoterapia domiciliare in cinque paesi europei: un'analisi comparativa. Mecosan 2000; 35: 137-148

3. Corsi F, Garattini L, Tediosi F. I dispositivi medici nei principali paesi europei. Milano, Edizioni Kappadue, 1999

4. Miselli V. Assistenza al paziente domiciliare. Roma, Il Pensiero Scientifico Editore, 1995

5. Rees PJ, Dudley F. Oxygen therapy in chronic lung disease. BMJ 1998; 317: 871-4

6. Lucioni C, Donner CF, De Benedetto F, et al. I costi della broncopneumopatia cronica ostruttiva: la fase prospettica dello Studio ICE (Italian Costs for Exacerbations in COPD). PharmacoEconomics - Italian Research Articles - 2005; 7(2): 119-134

7. Micheletto C, Pomari C, Righetti P, Dal Negro RW. A 2-year health economics survey on 61 subjects in telemetric LTOT: preliminary results. The European Respiratory Journal 1995; 7 (suppl 18) [abstract]

8. Lucioni C, Donner CF, De Benedetto F, et al. I costi della broncopneumopatia cronica ostruttiva in Italia. Presentazione della prima fase dello studio ICE (Italian Costs for Exacerbations in COPD). PharmacoEconimics - Italian Research Articles 2004; 6 (1): 5-14 